\title{
Variación de la capacidad antioxidante y vitamina $C$, en el liofilizado de la fruta del camu camu (Myrciaria dubia)
}

\section{Variation of the antioxidant capacity and vitamin $C$, in the freeze drying of camu camu fruit (Myrciaria dubia)}

\author{
Jaime Basilio-Atencio ${ }^{1, a, *}$, Roberto Dávila-Trujillo ${ }^{1, b}$
}

\begin{abstract}
${ }^{1}$ Facultad de Ingeniería en Industrias Alimentarias, Universidad Nacional Agraria de la Selva, Carretera Central km. 1.5; Tingo María; Huánuco, Perú.

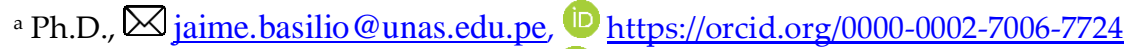

b Dr., \roberto.davila@ @otmail.com, (ID https://orcid.org/0000-0001-7555-6300

* Autor de Correspondencia: Tel. +51 968942048

http://dx.doi.org/10.25127/riagrop.20221.780
\end{abstract}

http://revistas.untrm.edu.pe/index.php/RIAGROP revista.riagrop@untrm.edu.pe

Recepción: 04 de noviembre 2021

Aprobación: 06 de diciembre 2021

Este trabajo tiene licencia de Creative Commons. Attribution-NonCommercial-ShareAlike $\quad 4.0$ International Public License - CC-BY-NC-SA 4.0

\section{Resumen}

Se determinó el contenido de vitamina $\mathrm{C}$ y la actividad antioxidante en pulpa de camu camu maduro y pintón, antes y después de la liofilización. Las operaciones previas sobre los frutos de camu camu, fueron: selección y clasificación, control de pesos, despulpado, separación de la cáscara, semilla y pulpa y licuado. La precongelación se realizó en un ultracongelador a temperatura de $-40{ }^{\circ} \mathrm{C}$. En estas condiciones se realizó la sublimación. La pulpa de camu camu tuvo un rendimiento de $68.38 \%$. El proceso de liofilización presentó una disminución en el peso hasta un $9.8 \%$. La pulpa liofilizada de camu camu en polvo presentó para el estado verde y maduro un valor de 11557.62 y $14557.62 \mathrm{mg}$ ácido ascórbico/100 g de pulpa respectivamente. En ambos casos, se incrementó su valor por la evaporación del agua que fue alrededor del $94 \%$. La pulpa liofilizada de camu camu en polvo presentó un incremento en la concentración de polifenoles totales y capacidad antioxidante tanto para el estado el estado verde y maduro, en ambos casos se debió por la evaporación del agua durante la liofilización. 
Palabras claves: Camu camu, liofilizado, Vitamina C, Polifenoles totales, Capacidad Antioxidante.

\begin{abstract}
The content of vitamin $C$ and the antioxidant activity were determined in pulp of ripe camu camu and semiripe, before and after lyophilization. The previous operations on camu camu fruits were: selection and classification, weight control, pulping, separation of the shell, seed and pulp, liquefied. The pre-freezing was carried out in an ultra-freezer at a temperature of $-40{ }^{\circ} \mathrm{C}$, under these conditions the sublimation was carried out. The pulp of camu camu had a yield of $68.38 \%$. The lyophilization process presented a decrease in weight up to $9.8 \%$. The freeze-dried pulp of camu camu powder presented for the green and ripe state a value of 11557.62 and $14557.62 \mathrm{mg}$ ascorbic acid/100g of pulp respectively, in both cases its value was increased by the evaporation of water which was around $94 \%$. The freeze-dried pulp of camu camu powder presented an increase in the concentration of total polyphenols and antioxidant capacity for both the green and ripe state, in both cases it was due to the evaporation of water during freeze-drying.
\end{abstract}

Keywords: Camu camu, lyophilized, Vitamin C, Total polyphenols, Antioxidant capacity.

\section{INTRODUCCIÓN}

El Perú posee una región amazónica que tiene grandes potencialidades en frutos tropicales no convencionales. Entre todos ellos, está el camu camu, que es una fruta muy apreciada por su alto contenido de vitamina $C$, por ende, de su capacidad antioxidante. Sin embargo, en los procesos de conservación tratadas por métodos tradicionales, generalmente, se degradan los metabolitos secundarios y cuando son cuantificados, como resultado se pierden estas propiedades.

El principal componente en la pulpa de camu camu es el ácido ascórbico, con un promedio de 2780 mg / 100 g de pulpa (Myoda et al., 2010; Nascimento, Boleti, Yuyama, \& Lima, 2013; Gonzales, 2007; Rodrigues et al., 2004) y una pequeña cantidad de ácido dehidroascorbico, que representa solo un $4 \%$ del total de la vitamina C (Amaral; Silva; Lopes, 2020 y Ramos et al., 2002). Las proteínas representan el $0.5 \%$, mientras que los otros constituyentes se encuentran en cantidades similares a otras frutas tropicales (Rodrigues et al., 2004).

Taylor (2001) señala que el camu camu posee propiedades astringentes, antinflamatorias y nutritivas. Sin embargo, la vitamina $C$ es menos estable que todas las vitaminas hidrosolubles, es lábil al calentamiento en presencia de oligometales como el cobre y en solución, se degrada. Además, el ácido ascórbico se oxida fácilmente en presencia de oxígeno y la rapidez de oxidación aumenta cuando se eleva la temperatura. Así mismo, se sabe que la pérdida del color característico del camu camu es ocasionada por la oxidación de las antocianinas.

El proceso de concentración por calor de la pulpa de camu camu ayuda a preservar mejor el potencial nutricional de este tipo de productos. Este proceso está basado en la reducción de la actividad de agua y, de este modo, prolongar la vida útil de los jugos de fruta (Gomes et al., 2005). El proceso de concentrado tiene un papel fundamental en la calidad de los alimentos. Los jugos de fruta contienen entre el 75 al $95 \%$ de agua. Su retiro reduce los costos de empaque, 
almacenamiento y transporte, que prolongan la vida útil del jugo de la fruta (Brazil et al., 2008; Gomes et al., 2005).

Durante el procesamiento clásico de jugos, como la pasteurización térmica y concentración por evaporización, suelen ocurrir pérdidas de compuestos nutricionales termosensibles tales como la vitamina C. Nuevos procesos debe ser investigados como alternativas al tratamiento térmico convencional (Rodrigues et al., 2004). El proceso de concentración al vacío es una técnica que permite concentrar jugos o pulpas como las del camu camu con menores pérdidas de sus nutrientes. Existen otros procesos para la concentración de alimentos líquidos, tales como el proceso de membrana que es ventajoso en comparación con la evaporización clásica, debido a la posibilidad de operar bajo condiciones suaves, sin afectar la termosensibilidad de algunas sustancias como las vitaminas (Rodrigues et al., 2004).

Imán et al. (2010) determinaron el contenido de vitamina $\mathrm{C}$ en diferentes partes del fruto (pulpa, cáscara y pulpa más cáscara) en cuatro estados de maduración: verde, pintón, maduro y sobremaduro. Los resultados indican que los mayores contenidos de vitamina $C$ se encuentran en la cáscara del fruto en estados de maduración sobremaduro y maduro. El contenido de vitamina $C$, según estados de maduración, se ajusta a una curva de regresión cúbica, tanto para pulpa, cáscara y pulpa más cáscara, con $87 \%$, $90 \%$ y $98 \%$ de efectividad, respectivamente. Cuando aumenta el estado de maduración, la vitamina $C$ se incrementa en $515.43 \mathrm{mg}$ en cada $100 \mathrm{~g}$ de muestra de cáscara, lo que indican que la vitamina $C$ se concentrada principalmente en la cáscara del fruto.
La liofilización es un proceso en el cual primero se congela el material, para luego retirar el agua por sublimación a presión reducida, hasta alcanzar valores de $5 \%$ de humedad o menores, con la disminución de las pérdidas de los componentes volátiles o termosensibles. La liofilización termina con la etapa de almacenamiento del producto en forma controlada (libre de oxígeno y de vapor de agua) y se logra, de este modo, que los productos tengan una larga vida de anaquel y retengan, en una alta proporción, sus características organolépticas, físicas, químicas y biológicas.

Por medio de la liofilización, se logra reducir las pérdidas de los compuestos responsables del sabor y el aroma en los alimentos en mayor proporción que otros sistemas de secado. Son además productos fácilmente reconstituibles. Por ende, se considera a la liofilización como una multioperación (Salomão-Oliveira et al., 2016).

La liofilización, como uno de los métodos más importante de secado de alimentos, permite conservar los componentes del alimento, desde sus características organolépticas, nutricionales y principios activos. Por ello, resulta necesario deshidratar al camu camu con esta tecnología. El camu camu, es una fruta natural no convencional amazónica que se caracteriza por su alto contenido y alta propiedad funcional. Cabe recalcar que la bioactividad de estos metabolitos secundarios es muy importante para el organismo humano porque estos actúan como "atrapadores" de radicales libres a nivel celular.

Muchos de estos compuestos pierden su poder activo por algún tratamiento mal ejecutado. Por lo tanto, es necesario utilizar operaciones que 
permitan mantener, cualitativa y cuantitativamente, estas propiedades beneficiosas hasta el momento de su consumo y pueda ser aprovechado muy bien por nuestro organismo. El objetivo de la investigación fue determinar las variables de liofilización, además de evaluar la pérdida de capacidad antioxidante y vitamina $C$, en el liofilizado de fruta de camu camu.

\section{MATERIALES Y MÉTODOS}

\subsection{Lugar de estudio}

El presente trabajo se realizó en los laboratorios de Análisis de alimentos, Ingeniería de Alimentos y en el Centro de Investigación y Desarrollo Biotecnológico de la Amazonía (CIDBAM) de la Universidad Nacional Agraria de la Selva, en la ciudad de Tingo María.

\subsection{Materia prima y reactivos}

Se utilizó frutos de camu camu en estado de madurez pintón y maduro, procedentes del mercado de frutas de la ciudad de Tingo María, región Huánuco.

Los reactivos empleados fueron: Acido oxálico (MerkKGaA), 2-6 - diclorofenol- indofenol (MerkKGaA), Ácido ascórbico (MerkKGaA), 2,2-Diphenyl-1-1picrilhydrayl (DPPH; Sigma Aldrich, USA), Folin-Ciocalteu (Sigma Aldrich, USA), Metanol QP (MerkKGaA)

\subsection{Métodos de análisis}

\subsubsection{Análisis fisicoquímico}

- pH, método 11,032 (AOAC, 1995).

- Sólidos solubles, refractómetro 934,14 (C) (AOAC, 1995).
- Acidez titulable, método 942,15 (A, a) (AOAC, 1995).

- Humedad, 925,10 (AOAC, 1995).

- Cenizas, 923,03 (AOAC, 1995).

- Ácido ascórbico, método reportado (GOKMEN, et al. 2001).

- Actividad antioxidante (BRAND WILLIAMS et al. 1995).

\subsubsection{Cuantificación de ácido ascórbico}

Se determinó cuantitativamente por el método espectrofotométrico, basado en la reducción del colorante 2-6 diclorofenolindofenol, por medio de una solución de ácido ascórbico, a una longitud de onda de $515 \mathrm{~nm}$, recomendado por Gokmen et al. (2001).

\subsubsection{Determinación de la capacidad antioxidante.}

Se utilizó el método espectrofotométrico, basado en una reacción con el 2,2 - Diphenil - 1 - picrilhidracil (DPPH). Las lecturas fueron a una longitud de onda de $515 \mathrm{~nm}$, según el método de Brand-Williams et al. (1995).

\subsection{Metodología experimental}

\subsubsection{Liofilización de la fruta de camu camu}

Los frutos de camu camu se obtuvieron del mercado de frutas de Tingo María. Para evaluar el rendimiento de sus componentes, se separó la pulpa, cáscara y semilla. Luego, para someterlo a la liofilización, los frutos de camu camu no sufrieron ningún tratamiento térmico porque todo fue natural a un estado fresco. Para ello, se realizaron las siguientes operaciones:

- Clasificación - Selección: se seleccionaron los frutos que estuvieron en un buen estado de sanidad y se clasificaron por tamaño y estado de maduración. 
- Pesado: Se pesaron para evaluar el rendimiento y balance de materia.

- Desinfectado lavado: Se lavaron para eliminar partículas extrañas.

- Pelado: Se realizó el pelado para separar la cáscara, semilla y pulpa.

- Acondicionado: Para obtener mayores áreas, en la operación de secado, la pulpa y la cáscara se mezclaron y se licuaron. Luego, se colocaron en platos de acero inoxidables en forma de láminas delgadas circulares de aproximadamente $0.5 \mathrm{~cm}$ de espesor, previamente pesadas. Luego se realizó un precongelado a $-30 \quad{ }^{\circ} \mathrm{C}$, en una ultracongeladora por 24 horas. Finalmente, se llevó la temperatura a $-40{ }^{\circ} \mathrm{C}$ para realizar la liofilización.

- Liofilizado: Se utilizó un liofilizador (Labotec, 01 JLG/12Fd, South África). El proceso de liofilización se realizó a una temperatura de $-40{ }^{\circ} \mathrm{C}$ por 24 horas, hasta una presión de $5 \mathrm{~Pa}$ y con una temperatura de la muestra de $20^{\circ} \mathrm{C}$.

- Envasado: Para controlar la higroscopicidad, las muestras liofilizadas se colocaron en una bolsa de polipropileno, para su análisis de vitamina $\mathrm{C}$, polifenoles totales y capacidad antioxidante.

\subsection{Diseño estadístico}

Para el análisis de varianza (ANVA), se utilizó los datos obtenidos durante la liofilización de los análisis correspondientes de la vitamina $\mathrm{C} \mathrm{y}$ capacidad Antioxidante y se aplicó el programa de Statgraphics Centurión XV.

\section{RESULTADOS Y DISCUSIÓN}

\subsection{Análisis fisicoquímico del camu camu en estado maduro}

En la tabla 1, se presenta los resultados de algunos componentes del camu camu.

Tabla 1. Composición química en pulpa de camu

\begin{tabular}{lc}
\multicolumn{1}{c}{ Comu } & \\
\hline Humedad (\%) & Estado Maduro \\
Acidez (ácido cítrico) (\%) & 85.27 \\
$\mathrm{pH}$ & 3.26 \\
${ }^{\circ} \mathrm{Bx}$ & 2.56 \\
Sólidos totales (g/kg) & 6.50 \\
${ }^{\circ} \mathrm{Bx} /$ Acidez (Relación) & 14.73 \\
\hline
\end{tabular}

Los metabolitos más importantes del camu camu son los compuestos solubles. En este caso, los frutos en estado maduro contienen mayor concentración de estos componentes, entre ellos la vitamina C. En este sentido, fue necesario medir los valores de humedad, acidez, $\mathrm{pH}$ y sólidos totales del camu camu. Estos resultados fueron similares a lo reportado por Gonçalves, Lajolo, \& Genovese (2010).

\subsection{Balance de materia del camu camu}

Se realizó un control de pesos en todo el proceso para obtener la pulpa de camu camu y proseguir la operación de liofilización. En este tema, existen varias operaciones a considerar y está en función al tipo de materia prima a utilizar. Por ello, se le llama operaciones de acondicionamiento de la fruta, que considera forma y tamaño de la muestra. En esta investigación, se ha considerado trabajar con pulpa refinada, de tal modo que las partículas de los frutos fueron los más pequeños posibles y las áreas de exposición para convertirse en polvo sean eficientes y en menor tiempo. 
En la figura 1, se presenta el flujo de operaciones del balance de materia, a partir de $100 \mathrm{~kg}$ de materia prima hasta la obtención de la pulpa de camu camu con un valor de $68.27 \mathrm{Kg}$ que se redujeron por efecto de la cáscara y semillas. Estos valores son muy similares a los obtenidos por Calvay (2006).

Posteriormente, se sometió al proceso de liofilización de $68.27 \mathrm{~kg}$ de pulpa refinada. Se obtuvo $6.69 \mathrm{~kg}$ de camu camu en polvo. Estos valores fueron similares a los reportados por Vega (2005).

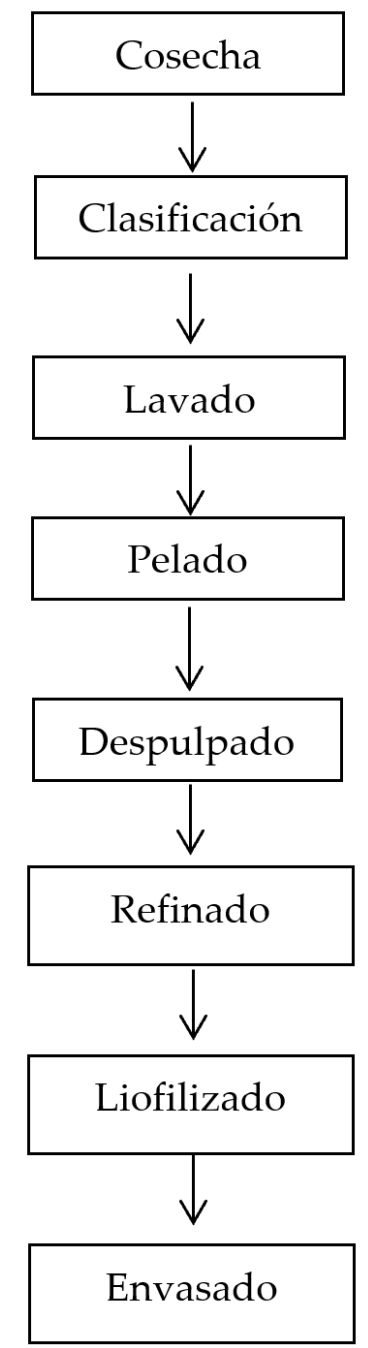

$100 \mathrm{~kg}$

$100 \mathrm{~kg}$

$99.85 \mathrm{~kg}$

$99.85 \mathrm{~kg}$

$68.27 \mathrm{~kg}$

$68.27 \mathrm{~kg}$

$6.69 \mathrm{~kg}$

$6.69 \mathrm{~kg}$

Figura 1. Flujograma del balance de materia durante la liofilización del camu Camu, en estado maduro.

\subsection{Rendimiento de los componentes del camu camu}

En la tabla 2, se puede apreciar el rendimiento de la cáscara, semilla y pulpa de camu camu. Estos valores resultan muy similares a los reportados por Calvay (2006).

Tabla 2. Rendimiento de los componentes del camu camu en estado maduro

\begin{tabular}{lc}
\hline Componente & Rendimiento (\%) \\
\hline Pulpa & 68.38 \\
Cáscara & 10.82 \\
Semilla & 12.80 \\
\hline
\end{tabular}

\subsection{Cuantificación de la vitamina $\mathrm{C}$, antes $\mathrm{y}$ después del liofilizado}

En la table 3, se presenta la variación del contenido de la vitamina $C$, antes y después del liofilizado de la pulpa de camu camu.

Los valores de la concentración de vitamina C variaron de acuerdo al estado de madurez. Como se observa, del estado pintón a maduro, se incrementó de 1256.26 a 1557.38 mg de ácido ascórbico/100 g, similar comportamiento fue reportado por Calvay (2006).

Durante la liofilización, se elevó la concentración de la vitamina $\mathrm{C}$, por efecto de la evaporación de agua que represento aproximadamente el $94 \%$. Estos valores, tanto para el estado pintón y maduro, variaron hasta 11557.62 y 14332.98, respectivamente, de manera similar a lo reportado por Vega (2005). 
Tabla 3. Variación de la vitamina $C$ durante la liofilización del camu camu ( $m g$ de ácido ascórbico/100g)

\begin{tabular}{lcr}
\hline Estado de Madurez & Estado fresco & Liofilizado \\
\hline Estado Pintón & $1256.26 \pm 6.43$ & $11557.62 \pm 59.15$ \\
Estado Maduro & $1557.38 \pm 6.02$ & $14332.98 \pm 55.36$ \\
\hline
\end{tabular}

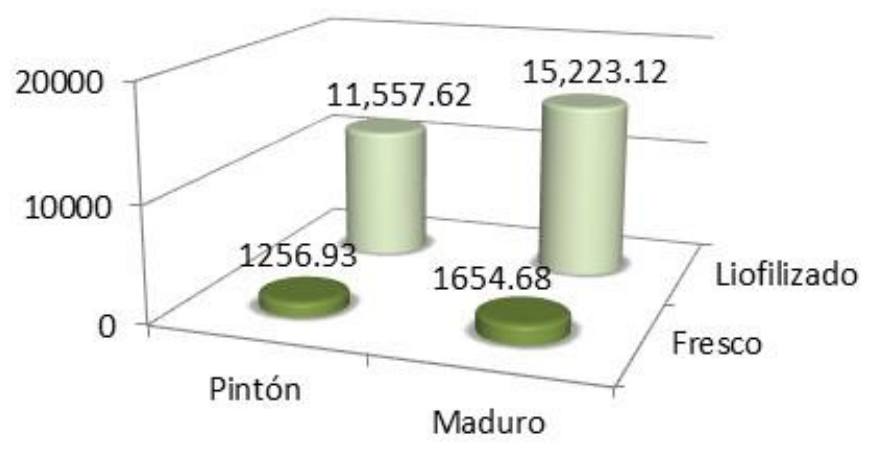

Figura 2. Variación de la vitamina C en pulpa fresca y liofilizada de camu camu.

\subsection{Cuantificación de los polifenoles totales antes y después del liofilizado}

En la tabla 4, se presentan la variación del contenido polifenoles totales antes y después del liofilizado de la pulpa de camu camu.

Tabla 4. Variación de polifenoles totales en liofilización del camu camu (mg catequina/g de pulpa)

\begin{tabular}{lcc}
\hline \multicolumn{1}{c}{$\begin{array}{c}\text { Estado de } \\
\text { Madurez }\end{array}$} & $\begin{array}{c}\text { Estado } \\
\text { fresco }\end{array}$ & Liofilizado \\
\hline Estado Pintón & $26.55 \pm 0.93$ & $244.32 \pm 8.60$ \\
Estado Maduro & $34.23 \pm 0.96$ & $314.97 \pm 8.85$ \\
\hline
\end{tabular}

Los valores de la concentración polifenoles totales variaron, de acuerdo al estado de maduración. Como se aprecia, del estado pintón a maduro, se incrementó de 26.5 a $34.23 \mathrm{mg}$ de ácido ascórbico/100 g. Un comportamiento similar fue reportado por Calvay (2006).

Durante la liofilización, se elevó la concentración de la vitamina $\mathrm{C}$, por efecto de la evaporación de agua, que representó aproximadamente el $94 \%$. Estos valores, tanto para el estado pintón y maduro, variaron hasta 244.32 y 314.97 respectivamente, de manera similar a lo reportado por Vega (2005).

Brazil et al. (2008) determinaron, para tratamientos de concentración de pulpa de camu camu, aumentos cuantitativos de la vitamina $\mathrm{C}$. 


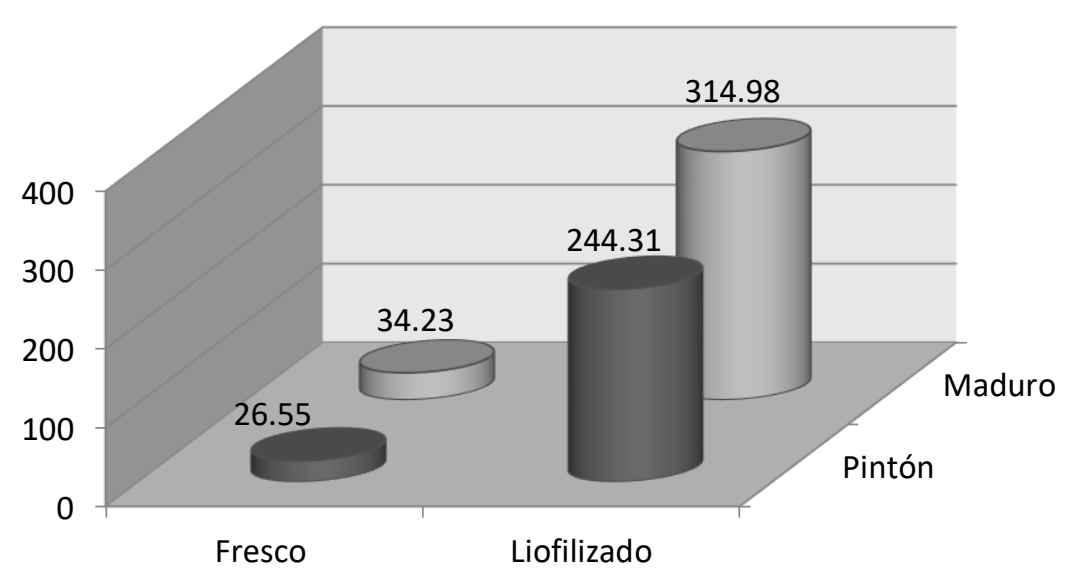

Figura 3. Variación del contenido de polifenoles totales en pulpa fresca y liofilizada de camu camu.

3.6. Cuantificación de la capacidad pintón a maduro, se incrementó de 1226.93 a antioxidante antes y después del $1654.68 \mathrm{mg}$ de ácido ascórbico/100 g y durante liofilizado la liofilización se elevó la capacidad antioxidante, por efecto de la evaporación de agua que represento aproximadamente el $94 \%$. contenido de capacidad antioxidante antes y después del liofilizado de la pulpa de camucamu.

Los valores de la concentración de capacidad antioxidante variaron de acuerdo al estado de Esto valores, tanto para el estado pintón y maduro, variaron hasta 11557.62 y 15223.11 , respectivamente, de modo similar a lo reportado por Vega (2005).

maduración. Como se evidencia, del estado

Tabla 5. Variación de la capacidad antioxidante en liofilización del camu camu ( $\mu$ g Eq. ácid. asc./g muestra)

\begin{tabular}{lcc}
\hline Estado de Madurez & Estado fresco & Liofilizado \\
\hline Estado Pintón & $1226.93 \pm 6.42$ & $11557.62 \pm 59.14$ \\
Estado Maduro & $1654.68 \pm 0.96$ & $15223.11 \pm 68.12$ \\
\hline
\end{tabular}

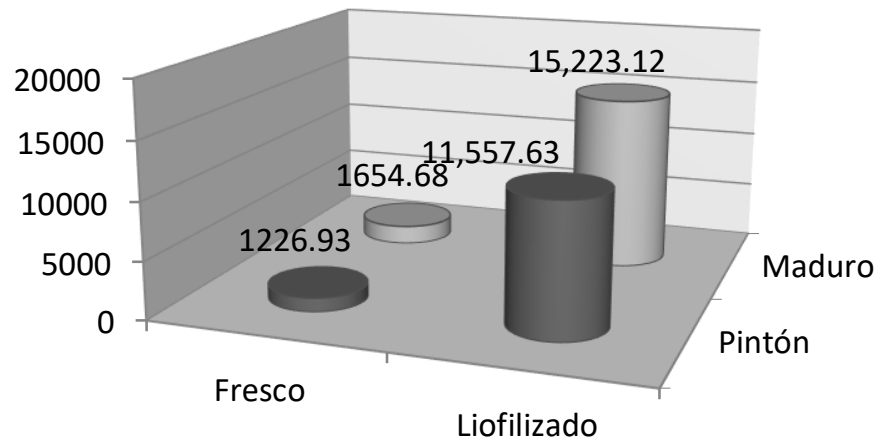

Figura 4. Variación de la capacidad antioxidante en pulpa fresca y liofilizada de camu camu. 


\section{CONCLUSIONES}

La pulpa de camu camu, en estado maduro, tiene mayor concentración de vitamina $\mathrm{C}$, con respecto al estado pintón.

El rendimiento de la pulpa, en comparación a la cáscara y semilla, estuvo en $68.38 \%$ y el balance de materia para la obtención de la pulpa para la liofilización fue de $68.27 \mathrm{~kg}$.

El rendimiento de la liofilización de la pulpa de camu camu, en estado maduro, fue de $9.8 \%$ y en el balance fue de $6.69 \mathrm{~kg}$ de camu camu en polvo.

La pulpa liofilizada de camu camu en polvo presentó, para el estado verde y maduro, un valor de 11557.62 y 14557.62 mg ácido ascórbico/100 g de pulpa, respectivamente. En ambos casos, su valor se incrementó por la evaporación del agua, que fue de alrededor del $94 \%$.

La pulpa liofilizada de camu camu en polvo presentó un incremento en la concentración de polifenoles totales y capacidad antioxidante, tanto para el estado verde y maduro, respectivamente. En ambos casos, esto se debió a la evaporación del agua durante la liofilización.

\section{Referencias}

Amaral, F.C., Silva, P.S. \& Lopes J.P. (2020). Vitamin characterization and volatile composition of camucamu (Myrciaria dubia (HBK) McVaugh, Myrtaceae) at different maturation stages. Food Sci. Technol, 41(4). https://doi.org/10.1590/fst.27120

AOAC. (1995). Official methods of analysis. Agricultural Chemicals Contaminants and Drugs.15으 Ed. Gaithersburg, Md. USA.

Brand-Williams, W., Cuvelier, M. \& Berset, C. (1995). Use of free radical method to evaluate antioxidant activity. LebensmWissTech., 28, 25-30.
Brazil, I., Teles, K., Oliveira, G., Maia, G. \& Figueiredo, R. (2008). Physicochemical changes during extraction and concentration of acerola juice (Malpighiaemarginata DC) using pectinases and clarifying agents. Braz. FoodTechnol., 10(4), 266-270.

Calvay, V. (2009). Evaluación de la capacidad antioxidante de camu camu Myrciaria dubia (H.B.K) Mc Vaugh. En dos estados de madurez en Tingo María. Tesis. Universidad nacional Agraria de la Selva. T.M. Perú. 122.p

Gokmen, V., Kaharaman, N., Dermir, N. \& Acar, J. (2001). Validación enzimática del método de cromatografía líquida para la determinación de ácido ascórbico y ácido dehidroascórbico en frutas y vegetales. B.V.J. Chromatogr., 881, 309-316.

Gomes, E., Mendez, S., Ereira, N. \& Barros, S. (2005). Evaluition of the acerola juice concentrated by reverse osmosis. Brazilian archivesof Biology and Tecology, 78, 175-183.

Gonçalves, F.M., Lajolo, M.I. \& Genovese. (2010). Chemical composition and antioxidant/antidiabetic potential of Brazilian native fruits and commercial frozen pulps. Journal of Agricultural and Food Chemistry, 58(8), 4666-4674

Gonzales, A. (2007). Frutales Nativos amazónicos, patrimonio alimenticio de la humanidad. Iquitos, Perú. IIAP. 76p.

Imán, C., Bravo, Z., Sotero, S. \& Oliva, C. (2010). Contenido de vitamina $C$ en frutos de camu camu Myrciaria dubia (H.B.K) Mc Vaugh, en cuatro estados de maduración, procedentes de la Colección de Germoplasma del INIA. Instituto de Investigaciones de la Amazonía Peruana - IIAP. Loreto, Perú.

Myoda, T., Fujimura, S., Park, B.J., Nagashima, T., Nakagawa, J. \& Nishizawa, M. (2010). Antioxidative and antimicrobial potential of residues of camucamu juice production. Journal of Food, Agriculture and Environment, 8(2), 304-307.

Nascimento, O.V., Boleti, A.P.A., Yuyama, L.K.O. \& Lima, E.S. (2013). Effects of diet supplementation with camu-camu (Myrciaria dubia HBK McVaugh) fruit in a rat model of diet-induced obesity. Anais da Academia Brasileira de Ciências, 85(1), 355-363.

Ramos, A., García, P. \& Pinedo, P. (2002). Evaluación de factores de procesamiento y conservación de pulpa de myrciaria dubia h.b.k. (camu-camu) que reducen el contenido de vitamina C (ácido ascórbico), Revista Amazónica de Investigación- Alimentaria, 2(2), 89-99.

Rodrigues, R., Menezes, H., Cabral, L., Dornier, M. \& Reynes, M. (2004). Evaluation of reverse osmosis and osmoticevapotation to concéntratecamucamu juice 
(Myrciaria dubia). Jurnal of food engineering, 63(1), 97102.

Salomão-Oliveira, A., De Souza, A., Cardoso, K., Pereira, T. \& Albuquerque, H. (2016). Ascorbic acid from lyophilized camu-camu fruit: stability and quality control of hard capsules. Rev Ciênc Farm Básica Apl., 37(1).
Taylor, L. (2001). Herbal Secrets of the Rainforest. Raintree Nutrition, Inc., Austin, Texas. USA. 78758. 360p.

Vega, V. (2005). Liofilización de pulpa de Myrciaria dubia hbk mc vaugh, camu camu. Folia amazónica, 14(2), 5156. 